\title{
HER2-amplified metastatic lung adenocarcinoma responds to fourth-line pyrotinib therapy: A case report
}

\author{
KAN GONG ${ }^{*}$, YI YANG ${ }^{*}$, HUAN HUANG, XUNJIE KUANG and XUEQIN YANG \\ Cancer Center, Daping Hospital, Army Medical University, Chongqing 400042, P.R. China
}

Received May 7, 2021; Accepted July 23, 2021

DOI: $10.3892 / \operatorname{mco} .2021 .2375$

\begin{abstract}
Despite the success of anti-HER2 therapy in patients with breast cancer with HER2 amplification or HER2 overexpression, the results of clinical trials on anti-HER2 therapy for lung cancer have not been satisfactory. The aim of the present study was to report a case of a non-smoker, female patient diagnosed with stage IIIA lung adenocarcinoma harboring HER2 amplification. The disease progressed despite surgery and multiple lines of chemotherapy, plus trastuzumab or lapatinib. The pan-ErbB inhibitor pyrotinib (400 mg/day) was commenced as a fourth-line regimen, and the patient achieved complete response with a time to progression (TTP) of 6 months. After the lung adenocarcinoma progressed, pyrotinib was continued, along with anlotinib and nivolumab. The patient achieved stable disease (SD) status with another 6 months of TTP. The overall survival of the patient was 28 months. Therefore, the present case suggests that the development of novel drugs may provide new and effective therapeutic regimens for lung cancer with HER2 amplification.
\end{abstract}

\section{Introduction}

Novel insights into gene mutations in lung adenocarcinoma have led to the molecular-stratified therapy of the disease. Fewer than $5 \%$ of patients with lung adenocarcinoma harbor HER2 (also known as ErbB2) alterations. Those with HER2 mutations have poorer survival outcomes compared with lung adenocarcinoma harboring other gene mutations, mandating

Correspondence to: Professor Xueqin Yang, Cancer Center, Daping Hospital, Army Medical University, 10 Changjiang Zhi Road, Yuzhong, Chongqing 400042, P.R. China

E-mail: yangxueqin@hotmail.com

*Contributed equally

Abbreviations: CR, complete response; $\mathrm{PR}$, partial response; $\mathrm{SD}$, stable disease; PD, progressive disease; TTP, time to progression; PFS, progression-free survival; CEA, carcinoembryonic antigen; NGS, next-generation sequencing; ORR, objective response rate; NSCLC, non-small lung cancer; OS, overall survival

Key words: NSCLC, HER2 amplification, pyrotinib tailored HER2-directed therapies in this subset of patients (1-3). The majority of HER2 mutations occur in HER2 exon 20 as a duplication or insertion mutation (4). The overall response rate of anti-HER2 therapy with HER2 inhibitors, including poziotinib, pyrotinib, and afatinib, ranges from 30 to $50 \%$ (5-7). In addition to HER 2 gene mutations, the mechanisms of HER2 activation include HER2 amplification or overexpression. Despite the success of anti-HER2 therapy in patients with breast cancer with HER2 amplification or overexpression, the results of clinical trials on anti-HER2 therapy for patients with lung cancer have failed (8).

The aim of the present study was to report a case of stage IIIA lung adenocarcinoma in a non-smoker female patient with primary chemoresistance who achieved partial response (PR) with anti-HER2 therapy using trastuzumab and lapatinib and complete response (CR) with pyrotinib after disease progression.

\section{Case report}

A 53-year-old Chinese, non-smoker, female patient with no family history of cancer was diagnosed pathologically with lung adenocarcinoma after undergoing thoracoscopic left upper lobectomy and hilar and mediastinal lymph node dissection at Daping Hospital (Chongqing, China) in March 2018. The TNM classification of the disease was T2N2M0 and stage IIIA. A CT scan after two cycles of pemetrexed $\left(500 \mathrm{mg} / \mathrm{m}^{2}\right.$, day 1) plus platinum $\left(75 \mathrm{mg} / \mathrm{m}^{2}\right.$, day 1 , every 3 weeks) revealed mediastinal lymph node and liver metastases. Magnetic resonance imaging of the brain revealed no intracranial metastasis. The CEA level increased to $180 \mathrm{ng} / \mathrm{ml}$ (normal reference value range: $0-5 \mathrm{ng} / \mathrm{ml}$ ). The patient was switched to a second-line regimen with albumin-conjugated paclitaxel $\left(260 \mathrm{mg} / \mathrm{m}^{2}\right.$, once every 3 weeks), platinum and bevacizumab $(7.5 \mathrm{mg} / \mathrm{kg}$, once every 3 weeks). However, the CEA level continued to increase $(414 \mathrm{ng} / \mathrm{ml})$. After one treatment cycle, the CT examination revealed a new liver lesion. Next-generation sequencing (NGS) of the surgical specimen revealed HER2 amplification (gene copy number: 11), HER2 mutation (1.46\%; F616L is not an exon 20 mutation), ErbB4 mutation (24.41\%), TP53 mutation (33.57\%), and other mutations (Table I). The tumor mutation burden was $17.9 / \mathrm{Mb}$. Supplementary immunohistochemistry (IHC) examination revealed HER2 overexpression (3+; Fig. S1). The third-line regimen was commenced in June 2018 with four courses of lapatinib 
$(1,250 \mathrm{mg} /$ day $)$ and one course of trastuzumab $\left(75 \mathrm{mg} / \mathrm{m}^{2}\right.$, once every 3 weeks), in addition to docetaxel $\left(75 \mathrm{mg} / \mathrm{m}^{2}\right.$, once every 3 weeks), platinum, vinorelbine $\left(25 \mathrm{mg} / \mathrm{m}^{2}\right.$, days 1 and 8 , every 3 weeks $)$ and capecitabine $\left(1,250 \mathrm{mg} / \mathrm{m}^{2}\right.$ twice a day, days 1-14, every 3 weeks). The patient achieved PR. The disease progressed with left axillary lymph node metastasis detected on CT scan in November 2018. The fourth-line regimen was then initiated, with pyrotinib $(400 \mathrm{mg} / \mathrm{day})$ combined with irinotecan $\left(220 \mathrm{mg} / \mathrm{m}^{2}\right.$, once every 3 weeks), and oxaliplatin $\left(130 \mathrm{mg} / \mathrm{m}^{2}\right.$, once every 3 weeks). After two cycles, the regimen was changed to pyrotinib monotherapy at $400 \mathrm{mg} / \mathrm{day}$. The patient achieved CR in February 2019. The metastatic foci in the liver and mediastinal lymph nodes disappeared, and the CEA levels returned to normal. The main treatment-associated side effect was grade 2 diarrhea.

The time to progression (TTP) of the patient was 6 months. The disease started to progress slowly, after six cycles of pyrotinib therapy. CT scans performed in April 2019 revealed new lesions in the liver, and the CEA level increased to $40 \mathrm{ng} / \mathrm{ml}$. The regimen was switched to afatinib (40 mg/day). After 1 month, the disease rapidly progressed with a marked increase in CEA $(639 \mathrm{ng} / \mathrm{ml})$ and CA125 $(1,255 \mathrm{U} / \mathrm{ml}$; cutoff value $<35 \mathrm{U} / \mathrm{ml}$ ) levels. A CT scan revealed slight progression in the lesions in the chest, but the liver lesions markedly progressed. A liver biopsy was performed in June 2019. NGS was performed using the same method as before. The results revealed HER 2 amplification (gene copy number: 23), and more gene mutations (Table II). No ErbB4 mutations were identified. The tumor mutation burden increased to $26.66 / \mathrm{Mb}$. Programmed death-ligand 1 (SP142) was negative as detected by IHC. As the results showed high copy number of HER2, high TMB, and high rates of chemotherapy-resistant gene mutations [such as KEAP1 (9) and NOTCH1 (10)], pyrotinib (480 mg/day) was continued, along with anlotinib (12 mg/day, days $1-14$, every 3 weeks) and nivolumab (3 mg/kg, once every 2 weeks). The disease remained stable until December 2019, and the patient achieved another 6 months of TTP. The patient eventually succumbed to the disease in July 2020 . The overall survival was 28 months. The changes in CT images and CEA levels over the course of treatment are shown in Figs. 1 and 2. The treatment regimens and responses are summarized in Table SI.

\section{Discussion}

Pyrotinib, a pan-ErbB blocker, has shown activity against EGFR $\left(\mathrm{IC}_{50}, 5.6 \pm 3.9 \mathrm{nM}\right)$, HER2 $\left(\mathrm{IC}_{50}, 8.1 \pm 2.3 \mathrm{nM}\right)$ and ErbB4 (11). By covalently binding with ATP-binding sites of intracellular kinase regions, pyrotinib inhibits the formation of homologous/heterodimer and auto-phosphorylation of HER family members, thus blocking the activation of the RAS/RAF/MEK/MAPK and PI3K/AKT signaling pathways and restricting tumor development (11). Based on the results of a phase II trial, the drug was conditionally approved in August 2018 in China for combination with capecitabine in patients with HER2-positive advanced or metastatic breast cancer previously treated with anthracyclines or taxane-based chemotherapy (12); however, it has not yet been approved by the US Food and Drug Administration. Second-line therapy with pyrotinib was found to be superior to lapatinib in patients
Table I. Gene mutational profile in a patient with lung adenocarcinoma by next-generation sequencing of DNA obtained from surgical specimens.

\begin{tabular}{lccc}
\hline Genes & Mutation sites & Frequency, \% & $\begin{array}{c}\text { Amplification } \\
\text { (copy no.) }\end{array}$ \\
\hline HER2 & F616L & 1.46 & 11 \\
ErbB4 & N280I & 24.41 & \\
GATA1 & S12P & 1.17 & \\
GNAS & T386P & 1.82 & \\
KIT & M1R & 2.58 & \\
KIT & A621S & 21.79 & \\
MSH3 & K281fs & 1.73 & \\
TP53 & G154V & 33.57 & \\
VEGFA & L139R & 1.08 & \\
\hline
\end{tabular}

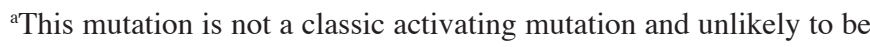
responsible for the responsiveness to HER2-targeted therapy.

Table II. Gene mutational profile of lung adenocarcinoma by next-generation sequencing of DNA from biopsy specimen after disease progression.

\begin{tabular}{lccc}
\hline Genes & Mutation sites & Frequency, $\%$ & $\begin{array}{c}\text { Amplification } \\
\text { (copy no.) }\end{array}$ \\
\hline HER2 & & & 23.69 \\
PIGF & & & 3.6 \\
ACAD5B & G360A & 12.61 & \\
AXIN1 & T332S & 39.39 & \\
ARID1A & P65Rfs*36 & 72.87 & \\
CD79A & W34R & 25.52 & \\
CDKN2A & $151-1 \mathrm{G}>\mathrm{T}$ & 50.78 & \\
CREBBP & $\mathrm{D} 539 \mathrm{G}$ & 19.31 & \\
FAT1 & $\mathrm{G} 1318$ & 24.81 & \\
FLT3 & $\mathrm{W} 105 \mathrm{C}$ & 23.4 & \\
IRF4 & $\mathrm{G} 314 \mathrm{~V}$ & 47.34 & \\
KIT & $\mathrm{A} 621 \mathrm{~S}$ & 36.94 & \\
KEAP1 & $\mathrm{R} 234 \mathrm{P}$ & 75.63 & \\
KMT2A & $\mathrm{G} 2409 \mathrm{~V}$ & 71.49 & \\
NOTCH1 & $\mathrm{P} 2199 \mathrm{~S}$ & 44.87 & \\
PIK3CA & $\mathrm{E} 218 \mathrm{Tfs} * 7$ & 13.17 & \\
PRKCI & I124L & 14.52 & \\
PRKCI & Y125C & 14.61 & \\
RPTOR & M280V & 35.67 & \\
TP53 & G154V & 67.9 & \\
WT1 & G216R & 57.51 & \\
\hline
\end{tabular}

with breast cancer previously treated with trastuzumab [progression-free survival (PFS): 18.1 vs. 5.6 months, respectively], probably due to the fact that pyrotinib targets more signaling molecules compared with lapatinib, which only acts on EGFR and HER2 $(13,14)$. However, to the best of our knowledge, whether pyrotinib is effective in patients with lung 

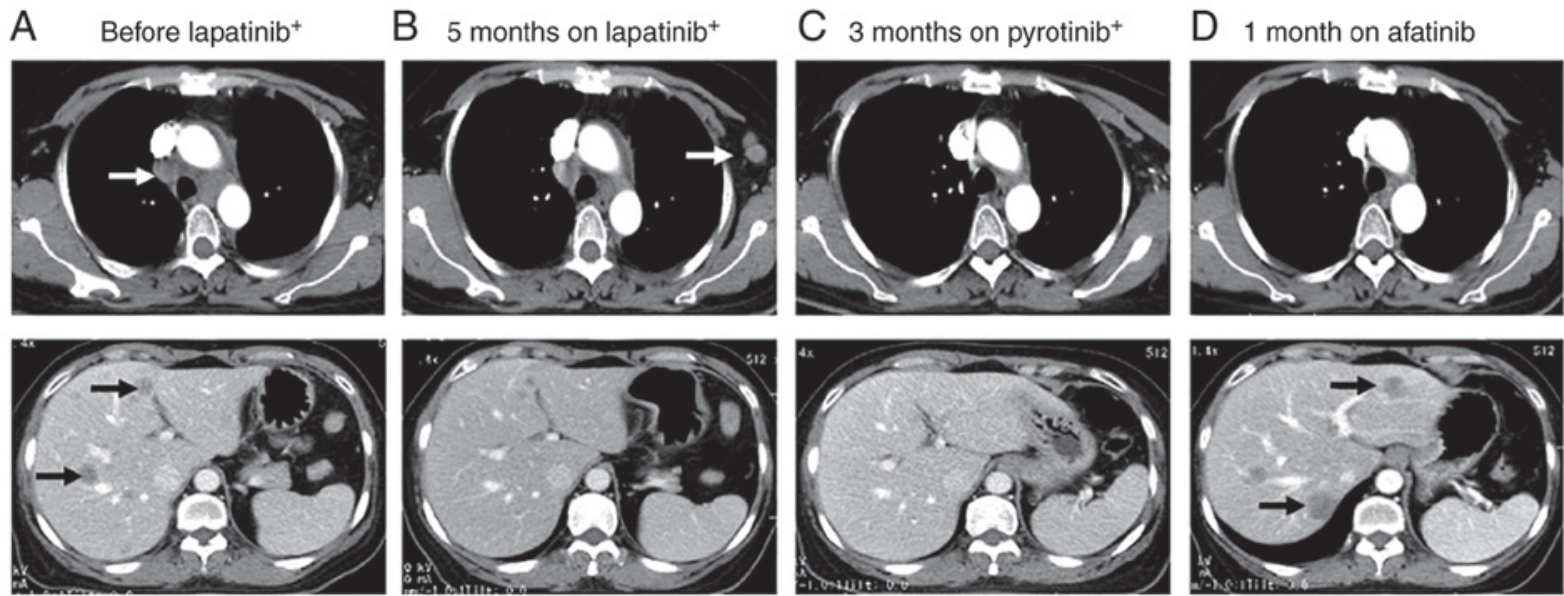

Figure 1. A 53-year-old female Chinese non-smoker was diagnosed with stage IV adenocarcinoma of the left lung with HER2 amplification on next-generation sequencing. CT images of the chest (upper panels) and upper abdomen (lower panels) are shown. (A) CT scan after two cycles of pemetrexed plus platinum revealed mediastinal lymph node metastasis and liver metastasis. After one cycle of albumin-conjugated paclitaxel, platinum and bevacizumab, the patient was treated with lapatinib plus chemotherapy. (B) The patient achieved partial response after treatment with lapatinib plus chemotherapy; however, 5 months later, the disease progressed with left axillary lymph node metastasis. (C) The patient achieved complete response 3 months after pyrotinib plus chemotherapy. (D) The disease started to slowly progress 6 months after pyrotinib therapy. The regimen was switched to afatinib; however, one month later, the disease progressed with the appearance of new hepatic lesions. Arrows, target lesions; +, plus chemotherapy.

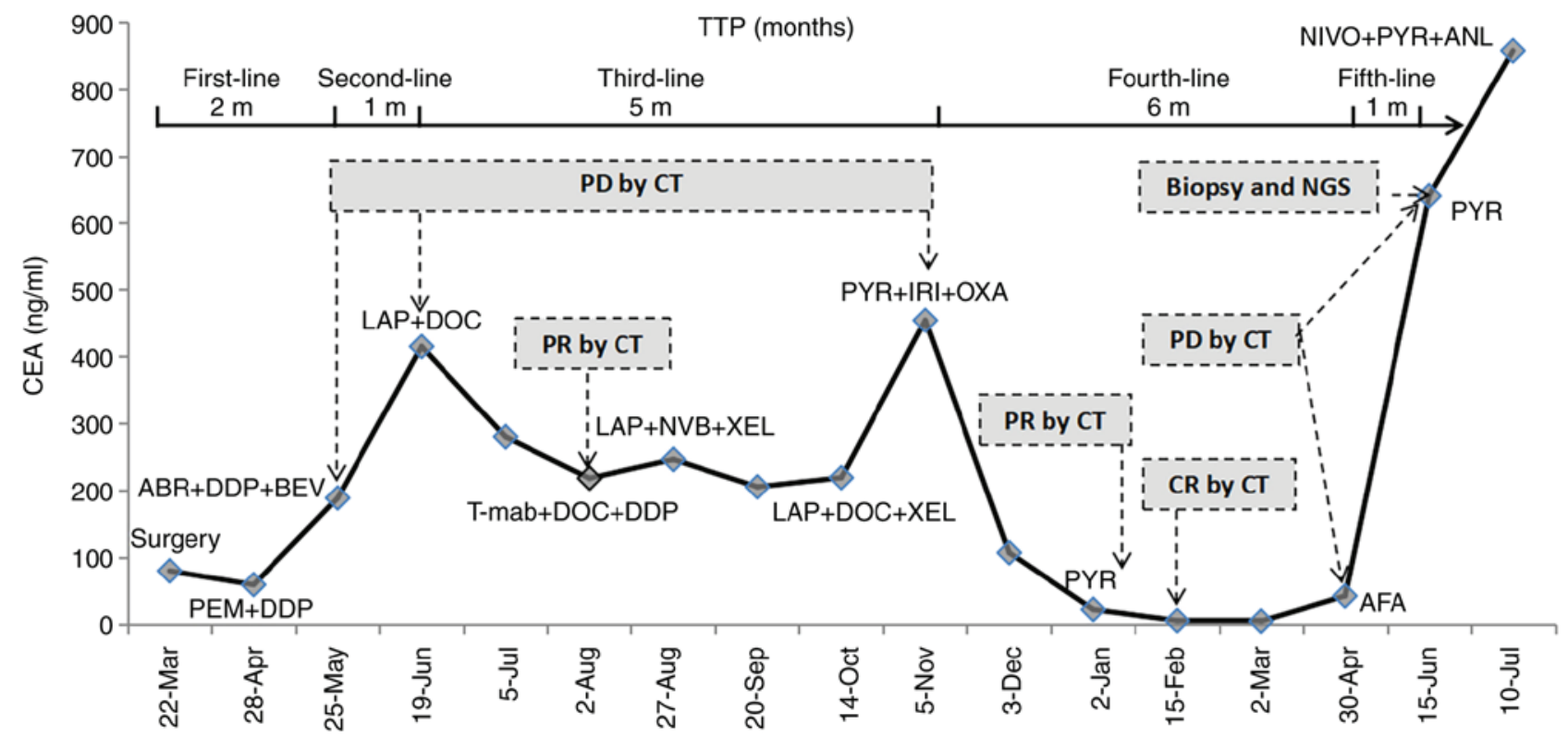

Figure 2. Changes of CEA levels over time. PEM, pemetrexed; DDP, cisplatin; ABR, abraxane; LAP, lapatinib; DOC, docetaxel; T-mab, trastuzumab; NVB, navelbine; XEL, xeloda; PYR, pyrotinib; IRI, irinotecan; OXA, oxaliplatin; AFA, afatinib; NIVO, nivolumab; ANL, anlotinib; TTP, time to progression; $\mathrm{PD}$, progressive disease; CR, complete response; PR, partial response; CEA, carcinoembryonic antigen.

cancer with HER2 amplification has not been reported to date. Experience from breast cancer has shown that some patients who were treated with trastuzumab or lapatinib developed primary resistance to anti-HER 2 therapy. Therefore, clinically, in patients with breast cancer, both trastuzumab and lapatinib are administered in combination with chemotherapy in most cases, except for trastuzumab monotherapy in the adjuvant setting. The present case had HER2 amplification with ErbB4 mutation. The patient also harbored a F616L mutation, which is not a classic activating mutation and is unlikely to be responsible for the responsiveness to HER2-targeted therapy. The patient was treated with four different HER2 inhibitors and at least five chemotherapeutic regimens. However, chemotherapy was ineffective during the first- and second-line treatment. The patient achieved PR with lapatinib, but developed primary resistance to trastuzumab. However, pyrotinib was effective, leading to $\mathrm{CR}$, particularly after resistance to lapatinib. Afatinib, which is also a pan-ErbB (EGFR/HER2/ErbB4) inhibitor, has been reported to be effective in a case of bladder cancer with HER2 amplification (15). After progression, the patient in the present case was switched to afatinib. However, the disease rapidly progressed, indicating that afatinib may not be suitable for patients resistant to pyrotinib. Diarrhea is the most common adverse effect observed with tyrosine kinase 
inhibitors targeting EGFR/HER2. The incidence of diarrhea in patients treated with pyrotinib plus capecitabine in breast cancer was $96.9 \%$, mainly grade $1-2$, with $15.4 \%$ of patients developing grade 3 diarrhea (14). The main adverse effect experienced by our patient was also diarrhea, which indicates that the adverse effects of pyrotinib in patients with lung cancer may be similar to those in patients with breast cancer.

HER2 amplification has been implicated as an oncogenic driver in lung cancers by The Cancer Genome Atlas (16). Although therapy targeting HER2 amplification or protein overexpression in lung cancer has long been explored, it is far less effective compared with breast cancer, and less effective compared with lung cancer with HER2 mutation. The objective response rate (ORR) of trastuzumab plus chemotherapy in lung cancer with HER2 amplification has been reported to be lower compared with that of chemotherapy alone $(17,18)$. Other new agents, including trastuzumab plus pertuzumab [ORR: $12.5 \%(2 / 16)$ ] (19) and trastuzumab emtansine [ORR: $0(0 / 8) ; 20 \%(4 / 20)](20,21)$ exhibited low efficiency in patients with HER2 amplification or overexpression [IHC (3+), FISH (+), or NGS (copy number increased)]. The pan-HER blocker dacomitinib was also found to be ineffective in lung cancer with HER2 amplification (0/4) (22). The use of other tyrosine kinase inhibitors, including lapatinib, niratinib, afatinib and pyrotinib, have not been reported in NSCLC. Anti-HER 2 therapy targeting HER 2 amplification in lung cancer has been characterized by low efficacy, for which there are possibly two main reasons: First, the HER2 signaling pathway in lung cancer is more complicated compared that in breast cancer. As HER2 does not have a known endogenous ligand for its extracellular domain, it activates downstream signaling pathways by forming heterodimers with other ErbB family receptors (23). Other ErbB family members (for example, ErbB4 mutation in this case) affect HER2 signaling, and targeting a single signaling molecule is not sufficient in lung cancer with HER2 amplification. Second, concurrent gene mutations in lung cancer contribute to the poor efficacy of HER2 therapy. In addition to HER 2 amplification, the present case had a TP53 mutation in the early stage of therapy and a KEAP1 mutation in the resistance stage. Concomitant TP53 or KEAP1 mutations have been reported in patients with EGFR mutations (24-26). The PFS of patients with these mutations receiving EGFR tyrosine kinase inhibitors was significantly shorter compared with that of patients without mutations. It was hypothesized that TP53 mutation contributed to the shorter TTP in our patient in the first- and subsequent second-line anti-HER 2 therapy. Thus, HER2 amplification or overexpression as a single parameter is an insufficient predictive biomarker in NSCLC.

There are currently no reports on the resistance mechanism of pan-ErbB blockers in HER2-amplified NSCLC. The resistance mechanism of lapatinib and niratinib in HER2-amplified breast cancer has been reported to be associated with bypass activation, such as HER 2 mutations and mutations in other ErbB family genes (27). In the present case, the inferred resistance mechanisms are as follows: The first is the insufficient ability to inhibit the HER2 signaling pathway, as the copy number of HER2 was higher than before and there was a mutation of PIK3CA. The second is bypass activation, which includes mutations in CDKN2A, Notch1 and KEAP1.
However, it does not appear to be associated with mutations in the ErbB family gene.

Our current case suggests that the development of novel drugs may offer new and effective therapeutic regimens for lung cancer with HER 2 amplification. Clinical trials or studies should be conducted to investigate the efficacy of novel therapeutic regimens for lung cancer with HER2 amplification.

\section{Acknowledgements}

Not applicable.

\section{Funding}

No funding was received.

\section{Availability of data and materials}

The datasets generated and/or analyzed during the current study are not publicly available due to protection of patient privacy and biosecurity reasons, but are available from the corresponding author on reasonable request.

\section{Authors' contributions}

KG and XY made the diagnosis and wrote the manuscript. YY, $\mathrm{HH}$ and $\mathrm{XK}$ created the tables and figures. KG and YY treated and followed up the patient, and they have also seen and can confirm the authenticity of all the raw data. All the authors have read, carefully revised and approved the final version of this manuscript.

\section{Ethics approval and consent to participate}

The present case report was approved by the Ethics Committee of Daping Hospital, Army Medical University, Chongqing, China [no. 2019(116)]. Written informed consent was obtained from the patient.

\section{Patient consent for publication}

Written informed consent was obtained from the patient for publication of the case details and any associated images.

\section{Competing interests}

The authors declare that they have no competing interests.

\section{References}

1. Arcila ME, Chaft JE, Nafa K, Roy-Chowdhuri S, Lau C, Zaidinski M, Paik PK, Zakowski MF, Kris MG and Ladanyi M: Prevalence, clinicopathologic associations, and molecular spectrum of ERBB2 (HER2) tyrosine kinase mutations in lung adenocarcinomas. Clin Cancer Res 18: 4910-4918, 2012.

2. Pillai RN, Behera M, Berry LD, Rossi MR, Kris MG, Johnson BE, Bunn PA, Ramalingam SS and Khuri FR: HER2 mutations in lung adenocarcinomas: A report from the lung cancer mutation consortium. Cancer 123: 4099-4105, 2017.

3. Li BT, Ross DS, Aisner DL, Chaft JE, Hsu M, Kako SL, Kris MG, Varella-Garcia $M$ and Arcila ME: HER2 amplification and HER2 mutation are distinct molecular targets in lung cancers. J Thorac Oncol 11: 414-419, 2016. 
4. Stephens P, Hunter C, Bignell G, Edkins S, Davies H, Teague J, Stevens C, O'Meara S, Smith R, Parker A, et al: Lung cancer: Intragenic ERBB2 kinase mutations in tumours. Nature 431: 525-526, 2004

5. Robichaux JP, Elamin YY, Vijayan RSK, Nilsson MB, Hu L, He J, Zhang F, Pisegna M, Poteete A, Sun H, et al: Pan-cancer landscape and analysis of ERBB2 mutations identifies poziotinib as a clinically active inhibitor and enhancer of T-DM1 activity. Cancer Cell 36: 444-57.e7, 2019.

6. Wang Y, Jiang T, Qin Z, Jiang J, Wang Q, Yang S, Rivard C, Gao G, Ng TL, Tu MM, et al: HER2 exon20 insertions in non-small-cell lung cancer are sensitive to the irreversible pan-HER receptor tyrosine kinase inhibitor pyrotinib. Ann Oncol 30: 447-455, 2019.

7. Peters S, Curioni-Fontecedro A, Nechushtan H, Shih JY,Liao WY, Gautschi O, Spataro V, Unk M, Yang JCH, Lorence RM, et al: Activity of afatinib in heavily pretreated patients with ERBB2 mutation-positive advanced NSCLC: Findings from a global named patient use program. J Thorac Oncol 13: 1897-1905, 2018

8. Oh DY and Bang YJ: HER2-targeted therapies-a role beyond breast cancer. Nat Rev Clin Oncol 17: 33-48, 2020.

9. Jeong Y, Hellyer JA, Stehr H, Hoang NT, Niu X, Das M, Padda SK, Ramchandran K, Neal JW, Wakelee H and Diehn M: Role of KEAP1/NFE2L2 mutations in the chemotherapeutic response of patients with non-small cell lung cancer. Clin Cancer Res 26: 274-281, 2020.

10. Yang LZ, Lei CC, Zhao YP, Sun HW, Yu QH, Yang EJ and Zhan X: MicroRNA-34c-3p target inhibiting NOTCH1 suppresses chemosensitivity and metastasis of non-small cell lung cancer. J Int Med Res 48: 300060520904847, 2020.

11. Ma F, Li Q, Chen S, Zhu W, Fan Y, Wang J, Luo Y, Xing P, Lan B, $\mathrm{Li}$ M, et al: Phase I study and biomarker analysis of pyrotinib, novel irreversible Pan-ErbB receptor tyrosine kinase inhibitor, in patients with human epidermal growth factor receptor 2-positive metastatic breast cancer. J Clin Oncol 35: 3105-3112, 2017.

12. Blair HA: Pyrotinib: First global approval. Drugs 78: 1751-1755, 2018.

13. Gourd E: Pyrotinib versus lapatinib in HER2-positive breast cancer. Lancet Oncol 20: e562, 2019.

14. Ma F, Ouyang Q, Li W, Jiang Z, Tong Z, Liu Y, Li H, Yu S, Feng J, Wang S, et al: Pyrotinib or lapatinib combined with capecitabine in HER2-positive metastatic breast cancer with prior taxanes, anthracyclines, and/or trastuzumab: A randomized, phase II study. J Clin Oncol 37: 2610-2619, 2019

15. Choudhury NJ, Campanile A, Antic T, Yap KL, Fitzpatrick CA Wade JL III, Karrison T, Stadler WM, Nakamura Y and O'Donnell PH: Afatinib activity in platinum-refractory metastatic urothelial carcinoma in patients with ERBB alterations. J Clin Oncol 34: 2165-2171, 2016.

16. Cancer Genome Atlas Research Network: Comprehensive molecular profiling of lung adenocarcinoma. Nature 511: $543-550,2014$
17. Langer CJ, Stephenson P, Thor A, Vangel M, Johnson DH and Eastern Cooperative Oncology Group Study 2598: Trastuzumab in the treatment of advanced non-small-cell lung cancer: is there a role? focus on eastern cooperative oncology group study 2598 . J Clin Oncol 22: 1180-1187, 2004.

18. Gatzemeier U, Groth G, Butts C, Van Zandwijk N, Shepherd F, Ardizzoni A, Barton C, Ghahramani P and Hirsh V: Randomized phase II trial of gemcitabine-cisplatin with or without trastuzumab in HER2-positive non-small-cell lung cancer. Ann Oncol 15: 19-27, 2004.

19. Hainsworth JD, Meric-Bernstam F, Swanton C, Hurwitz H, Spigel DR, Sweeney C, Burris H, Bose R, Yoo B, Stein A, et al: Targeted therapy for advanced solid tumors on the basis of molecular profiles: Results from mypathway, an open-label, phase IIa multiple basket study. J Clin Oncol 36: 536-542, 2018.

20. Hotta K, Aoe K, Kozuki T, Ohashi K, Ninomiya K, Ichihara E, Kubo T, Ninomiya T, Chikamori K, Harada D, et al: A phase II study of trastuzumab emtansine in HER2-positive non-small cell lung cancer. J Thorac Oncol 13: 273-279, 2018.

21. Peters S, Stahel R, Bubendorf L, Bonomi P, Villegas A, Kowalski DM, Baik CS, Isla D, De Castro Carpeno J, Garrido P, et al: Trastuzumab emtansine (T-DM1) in patients with previously treated HER2-overexpressing metastatic non-small cell lung cancer: Efficacy, safety, and biomarkers. Clin Cancer Res 25: 64-72, 2019.

22. Kris MG, Camidge DR, Giaccone G, Hida T, Li BT, O'Connell J, Taylor I, Zhang H, Arcila ME, Goldberg Z and Jänne PA: Targeting HER2 aberrations as actionable drivers in lung cancers: Phase II trial of the pan-HER tyrosine kinase inhibitor dacomitinib in patients with HER2-mutant or amplified tumors. Ann Oncol 26: 1421-1427, 2015.

23. Roskoski R Jr: Small molecule inhibitors targeting the EGFR/ErbB family of protein-tyrosine kinases in human cancers. Pharmacol Res 139: 395-411, 2019.

24. Labbé C, Cabanero M, Korpanty GJ, Tomasini P, Doherty MK, Mascaux C, Jao K, Pitcher B, Wang R, Pintilie M, et al: Prognostic and predictive effects of TP53 co-mutation in patients with EGFR-mutated non-small cell lung cancer (NSCLC). Lung Cancer 111: 23-29, 2017

25. Hou H, Qin K, Liang Y, Zhang C, Liu D, Jiang H, Liu K, Zhu J, Lv H, Li T and Zhang X: Concurrent TP53 mutations predict poor outcomes of EGFR-TKI treatments in Chinese patients with advanced NSCLC. Cancer Manag Res 11: 5665-5675, 2019.

26. Hellyer JA, Stehr H, Das M, Padda SK, Ramchandran K, Neal JW, Diehn M and Wakelee HA: Impact of KEAP1/NFE2L2/CUL3 mutations on duration of response to EGFR tyrosine kinase inhibitors in EGFR mutated non-small cell lung cancer. Lung Cancer 134: 42-45, 2019.

27. Xuhong JC, Qi XW, Zhang Y and Jiang J: Mechanism, safety and efficacy of three tyrosine kinase inhibitors lapatinib, neratinib and pyrotinib in HER2-positive breast cancer. Am J Cancer Res 9: 2103-2119, 2019. 